\title{
Elementos previos a la indagación sobre el pensamiento crítico en teoría lingiíística por parte de los licenciados en idiomas: el caso del programa de Lengua Gastellana
}

Resumen:

n el presente artículo expondremos en primera medida, las circunstancias que nos impulsaron a formular el proyecto de investigación. Para ello se comentan algunas experiencias que hemos vivido a lo largo de la carrera, además planteamos algunos interrogantes que se han suscitado a lo largo de nuestra formación. Seguidamente se presentará el marco teórico que hemos utilizado en la formulación de la herramienta el cual está compuesto por el pensamiento crítico, algunas caracteristicas que hacen a una persona o a un estudiante pensador crítico y la importancia de desarrollarlo en la formación de licenciados en lengua castellana. La segunda parte del marco teórico está referida a la importancia de establecer la forma en la enseñanza del licenciado, la relación entre los saberes disciplinares y pedagógicos y la manera de cómo evaluarse. La última parte referida al marco se dedica a tratar de esclarecer el objeto de estudio del licenciado en Lengua Castellana, alli se pone en tela de juicio los procesos teóricos y metodológicos que se enseñan en
Juan Camilo Puentes theramoness@gmail.com Anthony Forero A. antonycoa@gmail.com Karla Ibán̄ez Perdomo krlita11_29@hotmail.com Alexandra González Herrera aleja0592@hotmail.es Semillero de Investigación Sokal Grupo de Investigación IPPE lingüística para la enseñanza de la lengua castellana, además de la importancia del Pensamiento Crítico en el licenciado en formación. Finalmente se expondrá el proceso de la construcción de la herramienta y se expondrán las modificaciones que tuvo cada una de las actividades de la prueba y se argumentará lo que se pretendía lograr con ellas.

Palabras Claves: Discurso disciplinar y pedagógico, pensamiento crítico, transversalidad, evaluación e investigación.

\section{Contexto de la herramienta}

Considerando que la ciencia es hecha por seres humanos, y que estos están inmersos en diferentes realidades culturales, decir que esta depende absolutamente de elementos disciplinares, académicos y sobre todo objetivos (aspecto cuestionable desde las Ciencias Sociales) es una equivocación. Pues esta después de la segunda guerra mundial se ha convertido en algo relativo, inexacto y sobre todo parcial. Por eso los elementos extradisciplinares no son meramente 
circunstanciales, sino un elemento de crucial importancia en cualquier proyecto de investigación, sin importar el área de conocimiento que abarque.

Es por lo anterior que resulta vital comentar de manera sucinta de dónde y por qué surge el presente trabajo de investigación: $L a$ Transversalidad y el desarrollo del Pensamiento Crítico en cinco asignaturas del núcleo especifico de lingüistica del programa de Lengua Castellana. En nuestro proceso de desarrollo profesional como licenciado del programa de Lengua Castellana, hemos vivido diferentes circunstancias que llevaron a generar una serie de preguntas a partir de diferentes situaciones que son comunes en el diario vivir de nuestro proceso formativo, por ejemplo: muchos estudiantes del programa, durante los primeros semestres, empezaron a polarizar los diferentes saberes (lingüística, literatura y pedagogía) que hacen parte de ese proceso de desarrollo del licenciado en lengua castellana; es decir que unos (o casi todos) se querian convertir en literatos, críticos o poetas, otros se querían convertir en pedagogos y los últimos, quizás, estaban interesados en el estudio de los diferentes procesos de significación humana (i.e. la lingüística). Esta situación en el transcurso de los semestres se fue haciendo mucho más evidente, ocasionando altercados entre los mismos condiscípulos.

Además, estas dificultades se empezaron a intensificar por disputas personales que día a día se hacian más evidentes entre los mismos profesores, tanto del programa como catedráticos, pues sus apreciaciones personales ocupaban más tiempo que el discurso disciplinar; estas apreciaciones personales de los docentes en muchas ocasiones empezaron a influir de manera significativa en el proceso de formación como estudiante, pues de manera errónea uno empieza a relacionar las materias con la conducta personal de los profesores, esto quiere decir que uno resulta siendo víctima, en algún sentido, en su formación disciplinar por disputas extra-disciplinares de los formadores de formadores.

En ese transcurrir de los semestres nos empezamos a dar cuenta que esas disputas personales entre colegas van no solo acompañadas de esa generación de polaridad disciplinar entre los estudiantes, sino también en la misma manera como se imparten los cursos. Esos comentarios emotivos que surgen dentro de las clases donde un profesor advierte de los diferentes errores del otro, lo critica y lo ataca empieza a invadir sus diferentes líneas de trabajo disciplinar, que se transforman en poca rigurosidad académica y teórica, que se evidencia en bastantes casos en los cuales los estudiantes no pueden distinguir hasta dónde va la apreciación subjetiva del profesor y dónde aparece la labor académica.

Esto se puede ejemplificar con la siguiente anécdota: resulta que una vez en una clase, al terminar de dictar el tema, el profesor le preguntó a sus discipulos si tenian alguna pregunta. Uno de ellos levanto la mano y preguntó: "Profe y eso que usted me acaba de presentar ¿para qué me puede servir?" Pregunta de un estudiante de tercer semestre que indignó al maestro el cual le respondió, palabras más palabras menos: joven siéntase orgulloso de saber eso, con el solo hecho de que usted lo sepa ya es una persona diferente. Respuesta romántica del maestro, nada criticable, pero dada la naturaleza del aula de clase universitaria respuesta poco académica, respuesta que debió haber apuntado única y exclusivamente a las características formales del programa. Este comentario fue el detonante para que comenzaran a surgir diferentes preguntas que empezaron a poner en tela de juicio toda la formación que hasta el momento habiamos recibido y que dieron la iniciativa para investigar sobre la verdadera formación del licenciado. Esta preocupación sentó sus bases alrededor 
de quinto semestre y en cualquier lugar, menos en un aula de clase universitaria.

Este problema se convierte en algo crucial en circunstancias como la expresada por Eloísa Vasco cuando advierte: "no debe sorprender, que el maestro se apoye en procesos metodológicos de los cuales él tuvo experiencia como alumno. El maestro en formación aprende, no las formas que le enseñan desde planteamientos teóricos, sino las formas que más o menos inconsciente ( $y$ aun en contra de lo que hayan podido decir) utilizan o utilizaron sus maestros. La modificación de estas formas de enseñanza interiorizadas por imitación de los propios maestros es muy difícil, y cuando se da ocurre lentamente, y por lo general también por imitación de un modelo, pues cualquier propuesta de cambio crea mucha inseguridad"108.

Entendiendo esto, decidimos formular preguntas como ¿qué es verdaderamente necesario aprender para un licenciado en Lengua Castellana? ¿Existe un conocimiento básico en las licenciaturas? ¿Con qué criterios académicos y metodológicos se seleccionan los contenidos disciplinares impartidos en clase, o son solo mero gusto personal del docente? ¿Fomentan los docentes en sus discursos disciplinares una transversalidad disciplinar con miras al desarrollo del estudiante? Pero todo esto desembocó en un elemento que es el que nos lleva a generar la necesidad de formar un semillero de investigación: ¿Sirve todo lo enseñado en la formación como licenciado de Lengua Castellana? Y si es así, ¿para qué sirve? 2. Marco Referencial

A continuación se plantean los diferentes elementos teóricos que dan pie a la formulación de la prueba.

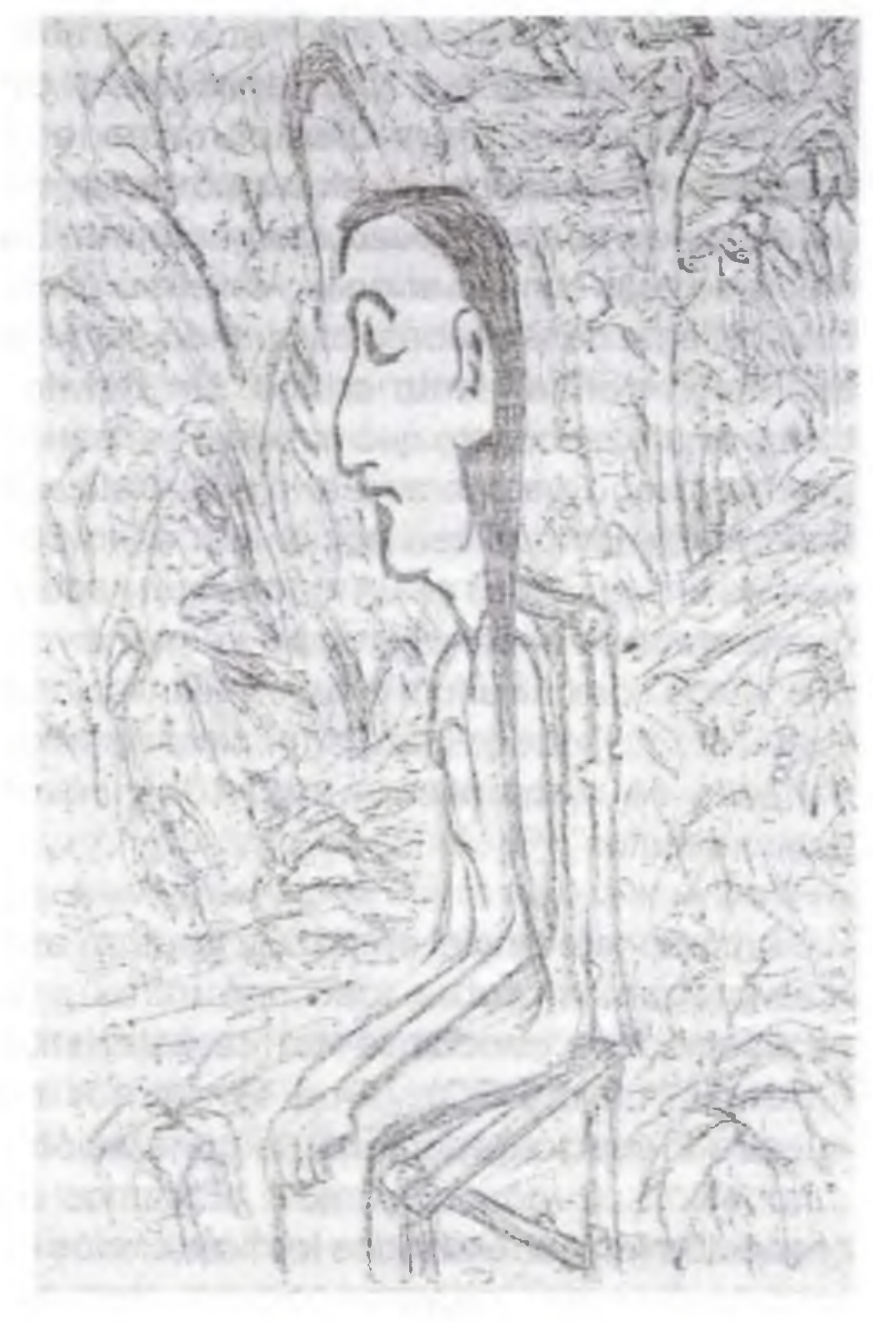

2.1 Nociones sobre el Pensamiento Crítico (PC) En medio de todo este mar de preguntas y de inconformismos empieza, de manera inconsciente, a surgir una especie de método de trabajo, que tiene como punto de partida la anterior cita de E. Vasco y que consiste en preguntar y cuestionar la manera en cómo se desarrolla nuestro proceso formativo desde la puesta en tela de juicio a los formadores de los futuros formadores, para asi ver desde nuestra realidad, entre nuestros condiscípulos, las diferentes problemáticas presentes.

\footnotetext{
${ }^{11}$ Eloisa, Vasco Montoya. El saber pedagógico. Razón de ser de la pedagogía. Pedagogía, Discurso y poder. Bogotá 1990
} 
Es entonces en medio de ese marco de traba jo, desarrollado sobre todo en cafés y en conversaciones extra-disciplinares con docentes interesados en el mismo. tema, en donde este trabajo empezó a tomar forma. En medio de esas conversaciones y en el momento más caliente de un debate es cuando surge la expresión pensamiento crítico. Un término bastante utilizado, pero que nos dejó pensando mucho. Luego, ya con asesoria especializada nos enteramos que esa noción tan usada en medios universitarios es un modelo muy actual que pertenece al paradigma de lo cognitivo y que "surge como una forma de reflexión racional e intuitiva $[. .$.$] que los seres humanos somos$ capaces de materializar en pos de nuevos conocimientos". ${ }^{109}$

Este marco referencial empezó a generar aun más preguntas, sobre todo con miras a la producción de conocimiento (característica fundamental del PC) ¿Qué elementos de nuestra formación apuntan a la generación y renovación del conocimiento? ¿Estamos en capacidad (en general todos los licenciados en formación) de generar algún tipo de conocimiento académico? Y los semilleros de investigación, ¿producen conocimiento, o son solo herramientas, 0 ayudantes de un gran proyecto de investigación coordinado por un maestro y del cual ellos no tienen ni idea? Y en el campo estrictamente académico y disciplinar ¿Qué aportan los estudiantes por ejemplo a la renovación curricular? ¿Son tenidos en cuenta los diferentes trabajos hechos por nosotros (en el caso de las tesis de grado, ensayos, documentos, etc.) en la construcción y desarrollo de los procesos académicos al interior de los programas académicos, de la Facultad y en últimas de la universidad?
En nuestra carrera se ha criticado mucho un material que se usa en los primeros semestres como guía para los cursos de lingüística: "Aproximación a las perspectivas teóricas sobre el lenguaje" de la profesora Mireya Cisneros y Omer Silva; este texto en su presentación plantea: "Nuestra intención desde el comienzo no fue hacer solamente registros descriptivos acerca de los planteamientos teóricos en torno al lenguaje, sino que pretendimos acompañar esta descripción con la reflexión, la contextualización y la relación en torno a los mismos con el fin de contribuir desde las aulas universitarias a la generación y transformación de saberes con fundamentos teóricos, para que los lectores no sólo sean depositarios de un conocimiento existente sino que la lectura de este libro motive para crear sus propias reflexiones $y$, por ende, a ser partícipes en la creación del conocimiento." 110 Pero el tan criticado y mal interpretado texto fue el que nos dio luces para preguntar por algo que parece banal y con respuestas al alcance de cualquiera y que muchos dan por hecho: ¿cómo se trabaja para aportar, desde las aulas de la USCO, a la generación y transformación de los saberes, y cómo se motiva y se dirige las creación de diferentes reflexiones para que nos hagan participes de la creación del conocimiento? Es más ¿los profesores de la universidad Surcolombiana aportan desde su labor docente a la creación del conocimiento?

Es desde ese momento cuando nos dimos cuenta que algo fallaba y que sobre eso nosotros podriamos decir algo, tomando como guía los planteamientos del pensamiento crítico, pues "[este] es un pensamiento que tiene propósito:

\footnotetext{
${ }^{109}$ José G. Altuve. El pensamiento critico y su inserción en la educación superior. Universidad de los Andes. Merida, Venezuela. 2010 . 10. ${ }^{110}$ Mireya Cisneros y Omer Silva, Aproximación a las perspectivas teóricas sobre el lenguaje (Colombia: Universidad Tecnológica de Pereira 2007. El resaltado es nuestro.
} 
probar un punto, interpretar lo que algo significa, resolver un problema." (Facione, 2007). Este marco referencial empezó a convertirse en algo bastante significativo, pues en cierto modo recoge muchos de los actuales problemas de la educación, el cambio de paradigma ${ }^{111}$ que diferentes autores proponen es dirigido desde los trabajos desarrollados en el PC, pues este prepara a los estudiantes en capacidades "que le permitan el acceso a cualquier conocimiento disciplinar con autonomía, calidad, criterio y argumentación necesaria para que dicho conocimiento no solo sea un cúmulo de información" 112 y que es uno de los principales objetivos a alcanzar en las actuales concepciones de educación.

Pero, ¿por qué tomar como punto de partida el PC?, por qué mejor no seguir los derroteros planteados por el estudiante Carlos Andrés Vargas en su trabajo de grado ${ }^{113}$, por qué mejor no mirar las caracteristicas de aquella cultura del pensamiento en la universidad, pues ese seria un gran ejercicio, el cual estamos seguros reprobariamos con las peores calificaciones. Pero resulta que el trabajo de nuestro compañero fue dedicado a la formación primaria y secundaria, y según Villiarini citado por José Altuve plantea que cada una de las etapas de formación tiene un fin, él "otorgaba al kínder y la primaria la tarea de propiciar el desarrollo de la intuición a la inducción; a la secundaria, el paso de la inducción a la deducción; a la universidad el paso de la deducción a la sistematización"114. Esa es la razón por la que se le agrega el adjetivo crítico, pues la universidad tiene, según este marco, la labor de informar para luego sistematizar la información, proceso que es individual por eso de los estilos y ritmos cognitivos y apuntan a la generación y producción de conocimiento, es decir que la tarea de la educación superior, como este lo indica debe ser de lo más alto y dar elementos para ello.

Para comenzar a pensar en el PC desarrollado durante el proceso de formación en el programa de Lengua Castellana en el componente especifico de la lingüística (y en general de todos) seleccionamos un nivel inicial en el que por lo menos exista sobre qué cosa pensar, es decir sobre los contenidos, tener una información que se pueda pensar críticamente, razón por la que el memorizar es punto de partida de todo lo demás. ¿Cómo se puede pensar críticamente sobre algo que ni siquiera se conoce? Además, este elemento de la memorización, tan estigmatizado por ser conductista y por pertenecer a la educación tradicional es el que permite pasar al desarrollo del pensamiento y luego pasarlo por el pensamiento crítico; "A veces el pensamiento crítico que se requiere es elemental y fundamental; por ejemplo al estudiar un tema existen conceptos fundamentales que definen el núcleo de la disciplina y para comenzar a apropiarlo, uno necesita dar voz a aquellos conceptos básicos -es decir, plantear con sus propias palabras, lo que significa el concepto, con el fin de detallar su significado, nuevamente, utilizando sus propias palabras para posteriormente dar ejemplos de dicho concepto en situaciones de la vida real [...] el aprendizaje por memorización se convierte en el recurso primario". ${ }^{115}$

\footnotetext{
"1'Sir Ken Robinson_Las escuelas matan la creatividad TED 2006, Tomado del video disponible en: http://www.youtube.com/watch?v=nPB$41 \mathrm{q} 97 \mathrm{zg}$

112 José G. Altuve, Pensamiento critico inserción en la educación superior. (Mérida, Venezuela) 2010

${ }^{113}$ Carlos Andrés, Vargas, Conocimiento y sistematización de estrategias que enseñan a pensar desde la educación elemental. Monografia

de grado, 2010, Universidad Surcolombiana, Facultad de Educación.

${ }^{114}$ José G. Altuve, Pensamiento crítico inserción en la educación superior. (Mérida, Venezuela) 2010, 05-18, El resaltado es nuestro.

${ }^{115}$ Richard Paul y Linda Elder, Estandares, Principios, Desempeño Indicadores y Resultados con una Rúbrica Maestra en el Pensamiento Critico, Fundación para el Pensamiento Crítico. 2005. 10
} 
Pero luego de memorizar ¿qué hace un pensador crítico? este hace útil la información, hace que esta cobre sentido haciéndola práctica, el estudiante con esta capacidad mas allá de ser una enciclopedia andante, sabe "qué hacer con la información, dónde informarse, cómo solucionar los problemas que a diario le acompañan, con seguridad y claridad conceptual"116 es decir logra criterio. Un pensador critico es "El tipo de persona [...] que siempre desea preguntar "¿Por qué?" o "¿Cómo?" o "¿Qué pasa si?"117 en otras palabras hacen preguntas procedimentales, les interesan los procesos más que los contenidos, pues tienen claro que estos ya existen, que pertenecen a otro nivel del desarrollo; en este momento es preciso que el lector lleve a cabo un ejercicio: Piense en los diferentes docentes que ha tenido, luego tome como referencia sus maneras de evaluar, cuando evalúa ¿Qué es lo que más le interesa a ese docente? ¿Qué le repitan lo que dijo en clase? ¿Pone ejercicios referentes a la teoría presentada? ¿Plantea diferentes casos y espera a que el estudiante les de una solución? ¿Pregunta por procedimientos? Si en sus evaluaciones imperaban las preguntas memorísticas, si en las evaluaciones le piden que repita lo mismo que se dijo en clase, si a este "maestro" no le interesan los procedimientos sino solo los resultados, este docente tiene muchos elementos que no permiten identificar un pensamiento crítico en su formación y por ende no les interesa desarrollarlo en sus estudiantes.

Lo anterior se puede traducir de la siguiente manera, un pensador crítico tiene como principal característica un espíritu crítico; pero "Tener espiritu crítico no significa que la persona sea siempre negativa ni excesivamente crítica de todo y de todos"118, o que pregunte por cosas obvias, o que justifique su flojera y poco interés en decir que como ese tema no le interesa no lo estudia, este espíritu "se refiere a la forma como usted enfoca los problemas, las preguntas, los asuntos. Es la mejor manera que conocemos de llegar a la verdad"119 ; esta persona pregunta siempre para construir, pregunta para que se arme el debate en torno a algo que para él es importante, "Los expertos usan la frase metafórica espíritu crítico en sentido positivo. Con ello quieren decir curiosidad para explorar agudeza mental, dedicación apasionada a la razón, y deseos o ansias de información confiable ${ }^{120}$. Podemos decir que otra característica de este pensador crítico y su espíritu es el hecho que reflexione sobre sus procesos, de su propio diario viviry que este sea punto de partida para tomar decisiones, "En otras palabras, hace un juicio reflexivo y con propósito sobre qué creer y qué hacer - precisamente el tipo de juicio que constituye el centro del pensamiento crítico"121 Podemos afirmar entonces que hace parte de esta lógica un pensamiento reflexivo, del que según Ignacio Laiton Poveda ${ }^{122}$ fue J. Dewey el primero en comentar, y quien dijo: "la mera sucesión de ideas o sugerencias constituye el pensamiento, pero no el pensamiento reflexivo, no la observación y el pensamiento dirigidos a una conclusión aceptable."

Luego de describir algunas generalidades

\footnotetext{
${ }^{116}$ Ignacio Laiton Poveda, Formación de pensamiento crítico en estudiantes de primeros semestres de educación superior, Revista Iberoamericana de Educación, Bogotá, Colombia. 2010. 1.

${ }^{117} \mathrm{Facione}, \mathrm{P}$. Critico: ¿Qué es y por qué es importante?, 7

${ }^{118}$ Peter A. Facione. Pensamiento Critico: ¿Qué es y por qué es importante? 2007. 7

${ }^{119}$ Facione, P. Critico: ¿Qué es y por qué es importante?, 8.

${ }^{120}$ Facione, P. Critico: ¿Qué es y por qué es importante?, 7

${ }^{12}$ 'Facione, P. Crítico: ¿Qué es y por qué es importante?, 11

${ }^{122}$ Ignacio Laiton Poveda, Formación de pensamiento crítico en estudiantes de primeros semestres de educación superior, Revista lberoamericana de Educación, Bogotá, Colombia. 2010.
} 
sobre los elementos a tener en cuenta para identificar un pensador critico, es necesario traer la visión ideal que propone P. Facione: "El pensador crítico ideal se puede caracterizar no solo por sus habilidades cognitivas, sino también, por su manera de enfocar y vivir la vida. Esta es una afirmación audaz. El pensamiento crítico va mucho más allá del salón de clase. Y muchos expertos temen que algunas de las experiencias escolares sean, en realidad, nocivas para el fomento y desarrollo de un buen pensamiento.

El pensamiento crítico apareció antes de que se inventara la escolaridad; yace en las raíces de la civilización misma. Es una piedra angular en el camino que la humanidad recorre desde el salvajismo bestial hacia la sensibilidad global".

Otra visión ideal del pensador critico la tomamos de José Altuve: "El pensador crítico ideal es una persona habitualmente inquisitiva, bien informada, que confía en la razón, de mente abierta, flexible, justa cuando se trata de evaluar, honesta cuando confronta sus sesgos personales, prudente al emitir juicios, dispuesta a reconsiderar y si es necesario a retractarse, clara con respecto a los problemas o las situaciones que requieren la emisión de un juicio, ordenada cuando se enfrenta a situaciones complejas, diligente en la búsqueda de información relevante, razonable en la selección de criterios, enfocada a preguntar, indagar, investigar $y$, además, persistente en la búsqueda de resultados tan precisos como las circunstancias y el problema o la situación lo permitan."

Luego de presentar lo que se entiende por pensador critico surge una pregunta (propia de un pensador critico): si el estudiante con este espíritu se pregunta por la utilidad del conocimiento, por el para qué sirve, es válido que surja la pregunta ¿y por qué es útil o para qué puede servir ser pensador critico? Facione pone en cuestión posibles respuestas: ¿Hará que obtenga mejores notas en la universidad? ¿Asegura a futuro un mejor puesto de trabajo? Pero el mejorar las calificaciones es bastante corto como para que sirva de justificación para adentrarse en un mundo tan difícil como el del pensador critico; ahora, el hecho de adoptar este estilo de vida hace que uno tenga criterio o no para continuar haciendo algo que no le gusta; estas dos respuestas son muy limitadas pues al ser esto un espíritu las supera y se sitúa en una manera de ver la vida y de entender el mundo, ser pensador crítico se convierte "[no en] un propósito importante, si no el más importante, de la experiencia de educación superior, ya sea a nivel técnico o universitario, es alcanzar lo que la gente ha llamado ina "educación liberal".[...] liberal en el sentido de "liberador". ¿Y quién se libera? ¡Usted!" 124

El hecho de ser pensador crítico tiene como objetivo principal el ser libre en el sentido de poder hacer lo que se quiera a partir de una serie de pensamientos razonados; respuesta un poco volátil pero argumentada, como ya se dijo anteriormente "El pensador crítico ideal se puede caracterizar no solo por sus habilidades cognitivas, sino también, por su manera de enfocar y vivir la vida"125. En términos generales $P$. Facione dice sobre la meta a alcanzar lo siguiente:

"Sí, la educación liberal es mucho más que el pensamiento crítico. Involucra la comprensión de los métodos, de los principios, de las teorías y de las maneras

\footnotetext{
${ }^{123}$ José G. Altuve El pensamiento crítico y su inserción en la educación superior. Universidad de los Andes. Merida, Venezuela. 2010.15

${ }^{124}$ Facione, P. Crítico: ¿Qué es y por qué es importante?, 17

${ }^{125}$ Facione, P. Critico: ¿Qué es y por qué es importante?, 8
} 


\section{PAIDEIASurcolombiama 11}

de adquirir el conocimiento que es propio de las diferentes esferas intelectuales.

Se da en ella el encuentro. con las dimensiones cultural, ética y espiritual de la vida. Comprende la evolución de la toma de decisiones de carácter personal hacia otra que tenga en cuenta un nivel de integridad de principios y de preocupación por el bien común y la justicia social. También, el darse cuenta de las maneras en que nuestras vidas se moldean por obra de fuerzas politicas, sociales, psicológicas, económicas, ambientales y físicas, tanto globales como locales. Además está el crecimiento que proviene de la interacción con nacionalidades, culturas, idiomas, grupos étnicos, religiones y niveles socioculturales diferentes de los propios." 126

2.2 El Objeto de Estudio del Licenciado en pedagogia de idiomas (lengua materna $o$ extrajera)

Después de haber presentado los diferentes elementos que dan pie a nuestra definición de pensamiento crítico, se hace necesario bajarlo al caso de la formación en Lingüística en los centros universitarios, y más precisamente a la formación en Lingüística para los docentes en proceso formativo en Lengua castellana, pero vale la pena aclarar que eso no limita a que lo planteado aqui sea llevado a los diferentes casos de desarrollo formativo en pedagogía en idiomas.

Luego de esta advertencia y continuando con lo planteado hasta aquí, surge un nuevo interrogante que será el punto de partida para el desarrollo de la prueba (otra pregunta típica de pensador crítico) ¿por qué se hace necesario para el licenciado en Lengua
Castellana (o estudiante de pedagogía en idiomas) desarrollar pensamiento crítico? Partamos del siguiente razonamiento: el licenciado se encuentra en una situación bastante difícil en términos sociológicos, pues al pertenecer al campo de las Ciencias Humanas y trabajar con seres humanos, y entendiendo que las dinámicas culturales desarrolladas por estos varian mucho, y más en estos tiempos en los que nada es lo que parece ser, el docente tiene una exigencia constante: el hecho de vivir actualizando su saber. Es por esta razón por la que entendemos la tarea del docente en lengua castellana no solo como una profesión (en la que se distingue el tiempo de trabajo vs el tiempo de descanso), sino como un estilo de vida; en los términos que maneja $\mathrm{N}$. Buenaventura ${ }^{127}$ no es una profesión del tiempo compartido, sino que es una labor del tiempo total, es decir que toca (más que obligación, por gusto y placer al conocimiento) vivir pendiente de lo pasa en el mundo, de lo que se hace en el común vivir de la ciudadanía.

Esto se hace aun más complejo en la circunstancias actuales, pues todas las orbes de producción humana (cultura) deben vivir en contacto permanente para ligar y establecer lazos entre los conocimientos humanos: "La mayor complejidad en la estructura del conocimiento contemporáneo, que según Edgard Morin solo puede ser asumida por el "pensamiento complejo", impone la interdisciplinariedad como la manera adecuada de dar respuesta a esa complejidad. "La supremacía de un conocimiento fragmentado según las disciplinas, nos dice Morin, impide a menudo operar el vínculo entre las partes y las totalidades y debe dar paso a un modo de conocimiento capaz de aprehender los objetos en sus contextos, sus complejidades, sus

\footnotetext{
${ }^{126}$ Facione, P. Crítico: ¿Qué es y por qué es importante?,18.

${ }^{127}$ Nicolas Buenaventura, La construcción del tejido social o la importancia de hablar mierda, Bogotá, 1995.
} 
conjuntos". La interdisciplinariedad implica complementariedad, enriquecimiento mutuo y conjunción de los conocimientos disciplinarios"128.

Por eso a la hora de seleccionar la docencia como profesión y estilo de vida, es conveniente escuchar la advertencia desalentadora que hace el profesor Álvaro Avendaño' ${ }^{129}$ : "antes de decidir ser docente piense que después de 30 años de servicios, aún te van a tratar con sospecha" y podemos llevar esto más allá de lo subversivo que tiene la labor docente (como ocurre en el contexto real de la cita) sino también a las diferentes juicios de valor en cuanto a su trabajo estrictamente académico (disciplinar) pues siempre existirá sobre él un signo de interrogación gigante; puede existir mucho respeto para un profesor que sea doctor, que conozca medio mundo, que tenga publicaciones, pero esa tarea de educador va hacer que siempre este en boca de todos y recibiendo ataques directos por su manera de enseñar y abordar un área disciplinar. ${ }^{130}$

Consideremos un caso hipotético, la formulación de una pregunta que debe hacerse todo aquel que se sienta afectado por esta temática: ¿Cuál sería el conocimiento básico, necesario y pertinente en lingüística para poder dar una clase de idiomas (ya sea lengua materna como extranjera) en un colegio? De los seis cursos que tomamos los licenciados en formación en lengua materna de lingüística ¿Cuáles son los elementos teóricos y metodológicos que surgen de cada uno de estos cursos para abordar las diversas temáticas de la lengua castellana en el colegio? Esto lleva a preguntarse de manera casi ontológica ¿Para qué sirve toda la teoria vista en esos seis cursos de lingüística? Digamos por ahora, y como lo pueden confirmar todos los docentes en ejercicio y los practicantes de Lengua castellana: ¡PARA NADA!

Esta situación ha llevado a que los estudiosos del lenguaje, y a que los diferentes autores y de manera casi intuitiva y sin reflexión por parte de los diferentes docentes, se halla dado por sentado que el pensar el papel que juegan los estudios sobre el significar en el aula de clase se reduce a un enfoque: El Comunicativo. Este, perteneciente históricamente a los estudios del trabajo textual y a los autores interesados en la teoría sobre lenguaje puesta en funcionamiento (lingüística funcional), ha reducido al estudioso del lenguaje dirigido a la educación a un simple animador de lecto-escritura. Esto se ha hecho una verdad irrebatible, de la que no se puede dudar, algo que todos los licenciados, no solo en castellano sino en idiomas, damos por sentado: el único enfoque en lingüística autorizado para entrar a un aula de clase y pensar que lo que alli se haga son los enfoques comunicativos.

El pensamiento crítico ayuda a eliminar esos prejuicios, ayuda a que la ciencia sea más abierta y suprime esas verdades que se dan por sentadas. Un pensador crítico no destruye totalmente lo que no entiende, es decir que si no comprende el para qué de los cursos teóricos de lingüística no los puede dar por eliminados. Pensemos entonces para qué

\footnotetext{
${ }^{128}$ Carlos Tünnermann Bernheim, El rol del docente en la educación superior del siglo XXI. El resaltado es nuestro.

${ }^{129}$ Álvaro E. Avendaño, Práctica pedagógica y saber disciplinar en la USCO, Paideia, N¹4, 2009, 7-13.

${ }^{130}$ Esta es la razón por la cual existen propuestas investigativas como esta, que elimina ese carácter casi mítico de los docentes y los ponemos en tela de juicio, se cuestionan y se les dan recomendaciones, casi de igual forma como un profesor asesor guia a sus practicantes durante sus primeras experiencias docentes. Esa es la belleza de esta profesión, que no existen verdades definitivas y las disputas argumentadas y las criticas están a la orden del dia.
} 
dedicar seis semestres de nuestras vidas al estudio de algo que ni siquiera los profesores con experiencia y en ejercicio entienden, y mucho menos aplican; pensar en esto es bastante importante pues uno de los puntos más inciertos en la formación docente es precisamente esta relación entre lo disciplinar y lo pedagógico, que podemos reescribir como la disputa entre la teoría y la práctica.

En esa búsqueda a una posible respuesta nos hemos encontrado con un texto de Suzan Francis Salazar en el que explica de manera corta una de las seis tesis de Shulman con miras a la formación docente: el conocimiento pedagógico del contenido ${ }^{131}$. En ella plantea que la mejor manera de comprender la relación entre lo pedagógico y lo disciplinar esta en un manejo profundo de la disciplina, en donde a partir del conocimiento de esta se esté en capacidad de saber metodológicamente y disciplinariamente qué es lo pertinente o no para llevar a clase (teniendo en cuenta las caracteristicas de contexto), al respecto dice Salazar citando algunos fragmentos de Shulman (1999): Esta relación [entre lo disciplinar y pedagógico] implica que para poder ejercer la docencia, se requiere "la transformación de lo comprendido" de determinado cuerpo disciplinar. O sea, la capacidad de enseñabilidad de determinado contenido descansa, entre otros, en "[...] el conocimiento profundo, flexible y cualificado del contenido disciplinar, pero además, en la capacidad para generar representaciones y reflexiones poderosas sobre ese conocimiento."' 132

Esto implica que el pensador crítico que se forma en Lengua Castellana en la universidad Surcolombiana por ejemplo, sea capaz de seleccionar de los diferentes paradigmas en lingüística, luego de la memorización, la interiorización, el desarrollo del pensamiento y en el momento en que llegue al nivel del pensar críticamente y busque la utilidad de eso a lo que dedicó tanto tiempo en los últimos años. Se espera que pueda construir una respuesta que contemple las diversas posibilidades que le ofrece cada uno de esos paradigmas, modelos o enfoques y pueda seleccionar de ellos los diferentes criterios que le sirvan para el desarrollo de diferentes actividades en la clase de la cual es responsable en el colegio. Por eso volviendo a Salazar, que se inspira en el modelo de Shulman, afirma que el manejo profundo de la disciplina le facilita al docente anticipar los componentes y relaciones del contenido que pueden presentar problemas para su comprensión. "Un buen manejo de la disciplina significa saber que algo es así y comprender el por qué de esta naturaleza, pero además saber bajo qué circunstancias se valida este conocimiento: "Esto será importante en las subsiguientes decisiones pedagógicas que consideren el énfasis curricular"'"' 133

Esto nos llevó a disminuir espacio y reflexionar sobre lo siguiente: podemos decir que la pedagogía y su materialización en las didácticas no resultan siendo el fin último de la formación disciplinar (o sea que preguntar: y ustedes ¿Cómo harian para llevar este texto a clase? Es un error garrafal, disciplinariamente) sino que podemos ver esto como un enfoque desde el cual se aborda el área disciplinar, podemos decir entonces que la enseñanza hace llevar las concepciones disciplinares a planos más productivos y guiados sobre todo a la aplicación de todo un corpus teórico que cobra vida cuando se pone en práctica, en este caso a

\footnotetext{
131 Susan Francis Salazar, El conocimiento pedagógico del contenido como categoria de estudio de la formación docente, Actualidades investigativas en educación, Vol. 5, N² 2, Costa Rica, 2005.

${ }^{131} \mathrm{~S}$. Francis Salazar, El conocimiento pedagógico del contenido..., 4

${ }^{132} \mathrm{~S}$. Francis Salazar, El conocimiento pedagógico del contenido..., 4

${ }^{133} \mathrm{~S}$. Francis Salazar, El conocimiento pedagógico del contenido..., 5
} 
través de la enseñanza. Desde esta perspectiva no existe una diferencia entre el objeto de estudio del Lingüista (como profesional en el área) al estudiante de Idiomas que toma cursos en lingüística como uno de los componentes de su formación, la diferencia se establece en el qué hacer o el para qué son útiles esos conocimientos (en el pensar críticamente), que para el caso de licenciado sería el de la enseñanza-aprendizaje de dicho idioma, que se transforma en cómo desarrollar las habilidades comunicativas necesarias para lograr desde la competencia lingüistica de cada estudiante una buena actuación.

Ahora, como se podrán dar cuenta, nuestra visión es un poco diferente en este sentido pues no nos basamos enteramente en el perfil dado por el programa si no que tomamos como perfil ideal lo planteado por $\mathrm{M}$. Mahecha quien al preguntarse "¿Qué esperamos que nuestro estudiante de pedagogía en idiomas sea capaz de hacer como producto de un curso de lingüística? Debe ser capaz de adquirir una actitud cientifica frente al lenguaje, lo que implica la eliminación de prejuicios comunes tales como la superioridad intrínseca de una lengua sobre la otra, la complejidad de una lengua, la corrección lingüística (el buen decir) y la primacia de to escrito sobre lo oral, entre otras tantas. Además, el profesor de idiomas debe comprender la estructura, la función y el funcionamiento del sistema lingüístico, detallando los diferentes planos que lo componen. Debe conocer las implicaciones socioculturales de la lengua, fruto del proceso de representación mental de las comunidades."134

Pero como nosotros no pertenecemos al CNA, ni podemos hacer sugerencias para realizar una reforma curricular, decidimos pensar en cómo metodológicamente, y partiendo de lo que se tiene curricularmente hablando, podemos llegar a ese perfil ideal que formulamos anteriormente. Para ello proponemos como primer nivel, para alcanzar este perfil ideal siquiera llegar al poco ambicioso, desactualizado y disciplinariamente incoherente perfil que tenemos actualmente, y con el cual funcionamos como programa de Licenciatura en educación básica con énfasis en humanidades y Lengua Castellana, y que al respecto del dominio disciplinar de la lingüistica propone lo siguiente: ${ }^{135}$

Explicará científicamente los hechos de la lengua.

Explicará científicamente las habilidades para la comprensión y producción de textos orales y escritos.

- Estará en capacidad de desarrollar la competencia comunicativa.

Favorecerá en los estudiantes la conciencia de sí mismos y el reconocimiento crítico del contexto.

Este es entonces el derrotero a seguir, y el cual utilizaremos como punto de llegada mínimo y básico del estudiante en pedagogía de los idiomas, pues si esto es lo que nos tiene funcionando legalmente y es a lo que se comprometen formar, veamos a ver qué pasa.

2.3 El rol del formador en los procesos de enseñanza/aprendizaje de una disciplina.

A partir de esa metodologia que creamos, de manera inconsciente y que fue anteriormente comentada, decidimos pararnos en el terreno del docente universitario y trabajar desde ahi, como punto de partida y apoyados en nuestra experiencia como estudiantes, para intentar

${ }^{134} \mathrm{Miguel}$ Ángel Mahecha, ¿Para qué enseñamos lingüística?, Paideia, N¹4,2010, 28.

${ }^{135}$ Cfr. Perfil profesional del licenciado en educación básica con énfasis en humanidades y lengua castellana. En: http://www.usco.edu.col pagina/lengua-castellana. La información contenida en los corchetes es nuestra. 
generalizar las dificultades de los formadores de los futuros formadores a la hora de desarrollar un proceso de construcción disciplinar en lingüistica para los pedagogos de los idiomas.

Comencemos por decir que la enseñanza/ aprendizaje de la lingüística, y sea dicho de paso de otras asignaturas, el saber disciplinar y pedagógico constituyen dos aspectos de unas misma actividad del desarrollo profesional tanto de los formadores de formadores como de los futuros licenciados, los futuros formadores. Es en este sentido que apunta Heles Contreras (citado por Mahecha): "El lingüista, en su trabajo, cumple una doble función, la de investigador (un rol exclusivamente intradisciplinar) y la de profesor (atendiendo a su carácter de 'anfibio cultural')" ${ }^{136}$

La importancia de evidenciar tanto los saberes disciplinares y pedagógicos conlleva a establecer un compromiso inicialmente sobre la claridad del objeto de estudio de la formación del licenciado, anteriormente descrito; en segundo lugar, sobre la pertinencia de la investigación en la orientación y desarrollo de una pedagogía desde las mismas disciplinas (en nuestro caso la Lingüística); en tercer lugar, la identificación del tipo de público al que está destinado los saberes específicos; y finalmente, la evaluación de dichos procesos de formación.

Identificamos en este último aspecto (la evaluación) su importancia en la constitución de los anteriores puntos mencionados. Sobre la incidencia de este, Nelson López, citado por Katerine Suárez, precisa:

"La Evaluación entendida como el proceso académico mediante el cual se constata el logro de avances y desarrollos formativos, resultados de los procesos curriculares y de la naturaleza de las prácticas pedagógicas de formación, y a la vez, factor de revisión y modificación de los mismos, se constituye en un proceso esencial en la constitución de identidades profesionales. No obstante, hoy se encuentra significativamente influenciada por una visión procedimental que la reduce la mayoría de las veces a un acto de mera y simple calificación, en lo que a la evaluación interna hace referencia." 137

Al concebirse una evaluación distante de la estrecha relación entre los saberes disciplinares y los pedagógicos es inevitable guiar la formación de los estudiantes hacia una actitud de fracaso investigativo que posibilite contextualizar los problemas de su profesión y proporcionar las herramientas adecuadas para resolverlos. Por ello es necesario tener en cuenta las siguientes preguntas propuestas por Licet K. Suárez:

"¿[...] el docente construye un esquema teórico/ metodológico que conduzca al estudiante a redescubrir sus procesos de cognición? [...] ¿Los profesores universitarios tienen definido su objeto de estudio? Es más, ¿llevan a cabo una constante actividad de investigación? ¿La transmiten y vivencian en sus cátedras? ¿La investigación, como miembros de una Facultad de Educación, integra saber disciplinary saber pedagógico?"138

Si la formación universitaria que recibe el estudiante de lengua castellana tiene estas dificultades (i.e. no tener claridad sobre el objeto de estudio de la formación del licenciado, no poseer una actividad investigativa sobre la pedagogía desde el mismo saber disciplinar,

\footnotetext{
${ }^{136}$ Miguel Á. Mahecha, ¿Para qué enseñamos lingüística?, 27.

${ }^{137}$ Licet Katerine, Suárez, ¿Es la pedagogia la herramienta de la práctica docente?, Revista Paideia Surcolombiana №. 14, (2009):16

${ }^{338}$ Suárez, ¿Es la pedagogia la herramienta de la práctica docente? 17-18.
} 
para generar un PC, entre otras.), ¿cómo exigir una calidad de formación de los estudiantes de las instituciones educativas de primaria y secundaria? En ese sentido el papel de la academia dentro de la sociedad está destinado a mejorar las condiciones de aprendizaje sólo a través de la actividad permanente de la investigación en el PC, sobre este punto el ex decano de la Facultad de Educación Lino Muñoz señala:

"Cada programa debe lograr una o varias especificidades, una estructura académica y orgánica bien planteada, coherente y lógica, que articule los saberes especificos $y$ pedagógicos en relación con el medio regional. Se debe buscar un punto de partida que integre $y$ articule las áreas de formación profesional y específica con las de formación pedagógica, científica y metodológica. Igualmente se debe incorporar la investigación a todo proceso curricular para que los resultados entren a intervenir los curriculos y a calificar al profesional en cuanto a profundidad teórica, consistencia metodológica y actitud práctica coherente, para generar un proyecto cultural no especulativo." 139

Estas reflexiones del estado del arte que se han tejido contribuyen a la fundamentación de la herramienta (la prueba de lingüística) en el sentido de (de)- mostrar cuán importante es dar claridad sobre el objeto de estudio en la formación del futuro licenciado (i.e. establecer la relación entre el saber pedagógico y disciplinar desde el PC), a su vez la manera en que debe abordarse la enseñanza del objeto de estudio y cómo evaluarse tal objeto de estudio. Esto favorece superar el complejo de inferioridad intelectual universitario dado que se integra a estudiantes y profesores en los procesos de investigación (i.e. reflexión y valoración) de la profesión. ${ }^{140}$

\section{Proceso de construcción de la prueba}

Ahora que nos acercamos al proceso de construcción de la herramienta debemos mencionar cómo va a comprobar nuestra hipótesis de trabajo: los estudiantes del programa de lengua castellana no alcanzan un nivel básico de pensamiento crítico ni dominio del discurso disciplinar en el componente específico de lingüística, lo cual demuestra la ausencia de la transversalidad, debido a que existe entre los docentes del área una serie de disputas extradisciplinares que afectan de manera directa a la formulación disciplinaria de los cursos, puesto que imperan más estas disputas personales que su interés real por formar un licenciado bien fundamentado disciplinariamente en el área.

Para tratar de comprobar esta hipótesis lo que se intentó en primera medida fue seleccionar el tipo de herramienta más conveniente para aplicar: la observación, la entrevista, la encuesta, la prueba escrita y oral. Al socializar los alcances y límites de las mismas se llegó a un acuerdo que el tipo de herramienta pertinente era la prueba escrita. Las razones a favor de ésta fueron: el ejercicio escrito permite la organización y precisión de las ideas; la sistematización de la prueba lleva a establecer criterios precisos de valoración objetiva: pertinencia de la respuesta con relación a lo que se pregunta o a la actividad que se propone; el tiempo de aplicación es reducido y la sistematización más precisa al

${ }^{139}$ Informe del decano de la Facultad de Educación Lino Muñoz (1999: 1-2). El resaltado es nuestro.

${ }^{140}$ Un ejemplo de esta actividad de cooperación es el trabajo de grado de Carlos Andrés Vargas Artunduaga (Universidad Surcolombiana: 2011): "Conocimiento y sistematización de estrategias que enseñan a pensar desde la educación elemental". Éste expone elementos sobre cómo generar estrategias que estimulen el pensamiento de la educación elemental. 
momento de formular la categorías de análisis.

Pensando en las modificaciones de la herramienta se determinó que debía tener una parte dedicada a la reflexión gramatical debido a que es un componente transversal en la lingüística. Sin embargo, consideramos que no era apropiado valorar un conocimiento específico, la gramática, porque la población a la que estaba dirigida la herramienta (estudiantes de lingüistica I) no tenia los conocimientos necesarios para responder. Por tal razón, decidimos proponer una segunda prueba referida a la gramática cuando el semestre haya avanzado más, lo que permitirá que los estudiantes, de lingüística I especialmente, tengan elementos para responder una prueba de gramática, ya que se supone que a partir de este curso se comienza a trabajar y/o a dialogar con aspectos gramaticales.

La primera prueba consistió en proponer una primera parte encaminada a las citas de reflexión teórica sobre lengua, lenguaje y habla. La segunda parte consistía en una reflexión teórica de qué es un modelo lingüístico y cómo se constituye. Además, estos modelos lingüísticos permitían identificar el objeto de estudio de la lingüística. Por otro lado, esta segunda parte contenía la aplicación de dicha reflexión teórica por medio de situaciones como por ejemplo el análisis lingüístico de un chiste, la explicación de la lengua, el lenguaje y el habla en un sordomudo, la utilidad de enseñar lengua materna a un hablante nativo.

Teniendo en cuenta los anteriores aspectos se determinaron criterios que permitirían tener la versión final de la herramienta, estos fueron: la herramienta debe ser estrictamente de reflexión teórica, lo que implicaba suprimir los ejercicios de aplicación debido a que la formación que recibimos no está orientada a resolver problemas del lenguaje (discapacidades físicas) sino a la lingüística aplicada a la educación; debe ser desarrollada por los estudiantes que estén cursando cualquiera de los cursos de lingüística I, II, III, IV y V, debe evidenciar saberes transversales y debe dar cuenta de proceso de construcción y maduración de las respuestas.

La prueba mantuvo en el transcurso de la modificación de la herramienta, lo cual ha sido las citas de Mahecha y Apresian, y las actividades relacionadas con el objeto de estudio de la lingüistica y sus modelos teóricos. Las cuales consideramos pertinentes y fundamentales para comprender el objeto de estudio de la lingüística. Debido que al comprender el discurso disciplinar en el cual existe una pluralidad de otras voces disciplinares habrá que tener en cuenta que este se divide en dos partes: uno dedicado a la fundamentación y uno dedicado a la aplicación del mismo a la profesión. Por el momento nos referiremos a la primera parte (i.e. la fundamentación del discurso). ${ }^{141}$

Los autores de las citas seleccionadas con respecto al objeto de estudio de la lingüística se trabajan en los cinco cursos de lingüística. Pasando a las actividades tenemos que decir que se mantuvieron porque estimamos que era fundamental que quienes estén preparándose como futuros licenciados en lengua castellana tengan claridad sobre estas, ya que son el eje transversal para comprender generalidades en lingüística y que el transcurso de la carrera se estará profundizando.

\footnotetext{
${ }^{141} \mathrm{C}$ fr. Gianinna Muñoz, Contrapuntos epistemológicos para intervenir lo social: ¿cómo impulsar un diálogo interdisciplinar?, Revista Cinta moebio [citado el 24 de marzo de 2012]: disponible en http://www.facso.uchile cl/publicaciones/moebio/40/munoz.pdf
} 
La primera actividad relacionada con los objetos de estudio y el perfil profesional que propone el programa de lengua castellana, pretendia que los estudiantes establecieran la relación de la disciplina con su futura labor docente, esto teniendo en cuenta los planteamientos del Pensamiento crítico donde se alude a la producción y renovación de conocimiento partiendo de los ya adquiridos en su proceso de formación. Lo que se busca es que los licenciados en formación reflexionen y contextualicen los planteamientos teóricos recibidos en su proceso. Además consideramos importante la concientización del papel del profesional en las Ciencias Sociales (para nuestro caso el licenciado de lengua castellana), como lo señala L. Rodríguez citado Gianinna Muñoz "Las Ciencias Sociales en general acuden al debate sobre el rol de las profesiones frente a los grandes problemas sociales en el contexto actual, reconociendo la insuficiencia de los conocimientos que cada disciplina parceladamente puede poner a disposición de la interpretación y actuación sobre éstos (Rodríguez 2008). ${ }^{142 "}$ En este sentido se hizo necesario que los estudiantes manifestaran la pertinencia de los modelos teóricos con miras al perfil profesional.

En la segunda se intentaba que los estudiantes identificaran los modelos a partir de la citas, así mismo un representante junto con los datos más relevantes del mismo, para poder observar la interiorización que realizan de los saberes de la lingüística y la relación de estos con su formación como licenciados; esto con el fin de que manifestaran el dominio disciplinar partiendo de elementos fundamentales con los deben contar para poder adentrase en el conocimiento lingüístico, ya que la memorización es un aspecto importante y básico, como referente para la apropiación de una nueva información, que posteriormente se convertirá en conocimiento.

En la tercera se buscaba que los estudiantes comprendieran la relación y distinción entre lenguaje, lengua y habla, suponiendo que contaban con un conocimiento básico sobre estos conceptos que son importantes dentro de la lingüística; debido a que el ser humano en su proceso de apropiación y transformación de la realidad requiere desarrollar las dimensiones cognitiva y comunicativa; esto visto desde el pensamiento crítico, implica que el futuro

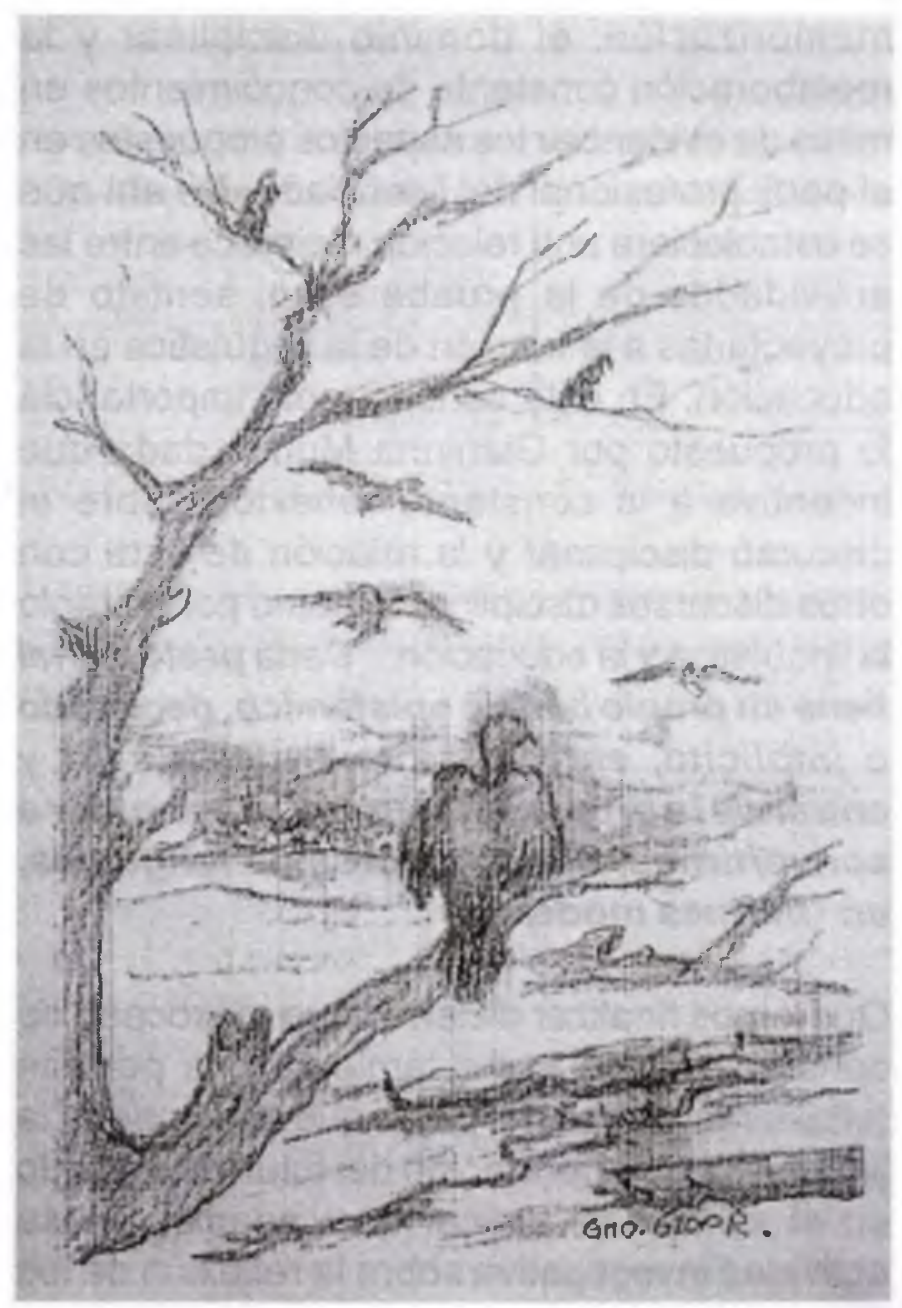

${ }^{142}$ Gianinna Muñoz, contrapuntos epistemológicos para intervenir lo social: ¿cómo impulsar un diálogo interdisciplinar? 91 
licenciado tome del abanico de subjetividades que le ofrece su realidad y reelabore conocimientos sustentados en elementos teóricos. Por tal razón, estas dimensiones son compresibles desde el punto de vista de los anteriores conceptos (lenguaje, lengua y habla) en el sentido de comprender y explicar la capacidad simbólica del ser humano a través de los conocimientos lingüísticos.

En cuanto a la última pregunta lo que se quería era ver cómo los estudiantes establecían la relación entre las citas seleccionas e identificaban el eje central entre las mismas, a partir de la puesta en función de la memorización, el dominio disciplinar y la reelaboración constante de conocimientos en miras de evidenciar los aspectos propuestos en el perfil profesional del licenciado. De ahí que se estableciera una relación reciproca entre las actividades de la prueba en el sentido de proyectarlas a la función de la lingüística en la educación. En este sentido toma importancia lo propuesto por Gianinna Muñoz dado que incentiva a la constante reflexión sobre el discurso disciplinar y la relación de este con otros discursos disciplinares como por ejemplo la lingüística y la educación: "Cada profesional tiene su propio bagaje epistémico, declarado - implícito, entonces, aprender a salir $y$ entrar de la propia episteme puede ayudara convertirnos en traductores de lenguajes, en "hermes modernos"." 143

Queremos finalizar diciendo que el proceso de construcción de la herramienta nos permite reflexionar acerca del papel que desempeña la lingüística en la formación del futuro licenciado en el sentido de indicar que, además de su actividad investigativa sobre la reflexión de los procesos de significación, este debe apropiarse de un marco epistémico que le muestre desde dónde leer y armar su propia taxonomía de saberes para intervenir en los procesos de enseñanza/aprendizaje.

\section{Bibliografía}

Altuve, José G. El pensamiento crítico y su inserción en la educación superior. Universidad de los Andes. Mérida, Venezuela. 2010.

Avendaño, Álvaro. Práctica pedagógica y saber disciplinar en la USCO, Paideia Surcolombiana, №14, (2009).

Buenaventura, Nicolás. La construcción del tejido social o la importancia de hablar mierda. Bogotá, Editorial Magisterio 1995.

Cisneros, Mireya y Silva, Omer. Aproximación a las perspectivas teóricas sobre el lenguaje. Pereira: Universidad Tecnológica de Pereira. 2007

Facione, Peter A. Pensamiento Crítico: ¿Qué es y por qué es importante? 2007.

Francis Salazar, Susan. El conocimiento pedagógico del contenido como categoría de estudio de la formación docente_Actualidades investigativas en educación, Vol $5, N^{\circ} 2$, Costa Rica, (2005)

Laiton Poveda, Ignacio. Formación de pensamiento crítico en estudiantes de primeros semestres de educación superior, Revista Iberoamericana de Educación, Bogotá, Colombia. 2010.

Mahecha, Miguel Ángel. "¿Para qué enseñamos lingüística?", Paideia Surcolombiana No. 14, (2009)

\footnotetext{
${ }^{143}$ Gianinna Muñoz, Contrapuntos epistemológicos para intervenir lo social: ¿cómo impulsar un diálogo interdisciplinar? 99. El resaltado es nuestro.
} 
Muñoz, Gianinna. Contrapuntos epistemológicos para intervenir lo social: ¿cómo impulsar un diálogo interdisciplinar?, Cinta moebio [citado el 24 de marzo de 2012]: disponible en http://www.facso.uchile.cl/ publicaciones/moebio/40/munoz.pdf

Paul, Richard y Elder, Linda. Estándares, Principios, Desempeño Indicadores y Resultados con una Rúbrica Maestra en el Pensamiento Crítico, Fundación para el Pensamiento Critico. 2005.

Sir Ken, Robinson. Las escuelas matan la creatividad TED 2006, Tomado del video disponible en: http://www. youtube.com/ watch? $v=n P B-41 q 97 z g$
Suárez, Licet K. ¿Es la pedagogia la herramienta de la práctica docente?, Paideia Surcolombiana No. 14, (2009)

Tünnermann Bernheim, Carlos. El rol del docente en la educación superior del siglo XXI.

Vargas, Carlos Andrés. Conocimiento y sistematización de estrategias que enseñan a pensar desde la educación elemental. Monografía de grado, para obtener el título de Licenciado en Lengua Española 2010, USCO. Vasco Montoya, Eloísa. El saber pedagógico. Razón de ser de la pedagogía. Pedagogía, Discurso y poder. Bogotá 1990. 


\section{UNWERSIDAD SURBOLOMBIANA FABULTAD DE EOIBABION}

Nombre:

Código:

Determinar el objeto de estudio de la lingüística es bastante difícil, asi se ha evidenciado en el Curso de Lingüística General (1915): "¿Cuáles el objeto a la vez integral y concreto de la lingüistica? La cuestión es particularmente dificil; [...] Otras ciencias operan con objetos dados de antemano y que se pueden considerar en seguida desde diferentes puntos de vista. No es asi en la lingüística. [...] Lejos de preceder el objeto al punto de vista, se diría que es el punto de vista el que crea el objeto, $y$, además, nada nos dice de antemano que una de esas maneras de considerar el hecho en cuestión sea anterior o superior a las otras. [...]Así, pues, de cualquier lado que se mire la cuestión, en ninguna parte se nos ofrece entero el objeto de la lingüistica". A partir de lo anterior, contemplemos diferentes visiones, planteamientos de ese objeto de estudio:
"A nuestro parecer, no hay más que una solución para todas estas dificultades: hay que colocarse desde el primer momento en el terreno de la lengua y tomarla como norma de todas las otras manifestaciones del lenguaje. En efecto, entre tantas dualidades, la lengua parece ser lo único susceptible de definición autónoma [...]" (SAUSSURE, 1916. PÁG. 33)

"La lingüística se ocupa del fenómeno que constituye el lenguaje $y$, por supuesto, sin descuidar la porción del lenguaje que se transforma en texto [...] la lingüística, por su parte -y es lo que la diferencia de toda otra disciplina cientifica-, se ocupa de una cosa que no es objeto ni sustancial en el lenguaje, ¿qué es lo que hay? Los datos del lenguaje no existen sino por sus diferencias, no valen más

\footnotetext{
${ }^{144}$ La versión final de nuestra prueba tuvo como referente de reflexión otra prueba titulada Quiz de lingüistica general: breve relato de una experiencia pedagógica en el aula. Elaborada por los profesores Joselyn Corredor Tapias y Cesar A. Romero Farfán. Esta prueba recogía el trabajo de socialización que tuvieron los estudiantes de la UTP en la cátedra de lingǘstica general durante el primer semestre de 2009 . El diseño de la prueba fue escrita y de selección múltiple, de ahi que el análisis de los resultados estuvieran orientados con un predominio cuantitativo que para los autores permitiría identificar que los rendimientos de los estudiantes obedecian a una adecuada intervención pedagógica. Por tal razón la prueba, como verá el lector, se fundamentó en el aspecto cualitativo. La prueba de los profesores Joselyn y Cesar está disponible en: http://dialnet.unirioja.es/servlet/articulo?codigo=3322169
} 
que por sus oposiciones." (BENVENISTE, 1979, pág. 32,34)

"[La Lingüistica] es la ciencia que estudia desde todos los puntos de vista posibles el lenguaje humano articulado, en general y en las formas especificas en que se realiza, es decir, en los actos lingüisticos y en los sistemas de isoglosas que, tradicionalmente o por convención, se llaman lenguas." (COSERIU, 1983, pág. 7)

"[...] El lingüista indaga sobre el hablar, sobre el significar. Podria decirse entonces que el objeto del lingüista son las unidades que generan sentido" (MAHECHA, 2011, pág. 20)

"La razón para concentrarse en el estudio de los actos de habla es, simplemente, esta: toda comunicación lingüistica incluye actos lingüísticos. La unidad de la comunicación lingüistica no es, como se ha supuesto generalmente, el simbolo, palabra, oración [...] sino más bien la producción o emisión del simbolo, palabra u oración al realizar el acto de habla. Considerar una instancia como un mensaje es considerarla como instancia producida y emitida. Más precisamente, la producción o emisión de una oración- instancia bajo ciertas condiciones constituyen un acto de habla y los actos de habla [...] son las unidades básicas o mínimas de la comunicación lingüística." (SEARLE, pág. 26)

"[...] La diversidad de la conducta humana, su adecuación a situaciones nuevas y la capacidad del hombre para innovar- el aspecto creador del uso del lenguaje proporciona la indicación principal de esto-[...] de este modo, una psicologia totalmente adecuada requiere la postulación de un "principio creador" junto con el "principio mecánico"" (CHOMSKY, 1974, pág. 25)
Actividades

1. Seleccione tres de las anteriores citas e ilustre la pertinencia de los objetos de estudio (modelos), teniendo en cuenta el perfil profesional del programa de LC.

2. Ahora: "Según Morales (1995, pp. 119-120) [Apoyado en Apresian dice]: un modelo no es más que un dispositivo teórico mediante el cual se logra progresivamente un dominio conceptual de la realidad. Siempre se construye un modelo de un objeto para lograr un conocimiento más profundo de dicho objeto [...] el modelo construido no entra en conflicto con lo que representa [...]".

Seleccione un modelo teórico del siglo $\mathrm{XX}$, el cual esté relacionado con una de las anteriores citas e identifique su autor (datos biográficos), tesis, argumentos, conclusiones, bibliografía (textos fundamentales).

3. Seleccione la(s) cita (s) que considera le permiten establecer (hacer explicito) una relación y distinción de los conceptos de lenguaje, lengua y habla.

4. Redacte un texto en donde identifique (distinga) la idea central que atraviesa todos los documentos 